\title{
Quercetin reverses the doxorubicin resistance of prostate cancer cells by downregulating the expression of c-met
}

\author{
YAN SHU $^{1}$, BO XIE ${ }^{1}$, ZHEN LIANG $^{2}$ and JING CHEN ${ }^{1}$ \\ ${ }^{1}$ Department of Urology, Tongde Hospital of Zhejiang Province, Hangzhou, Zhejiang 310012; ${ }^{2}$ Department of Urology \\ Surgery, First Affiliated Hospital, School of Medicine, Zhejiang University, Hangzhou, Zhejiang 310003, P.R. China
}

Received August 29, 2016; Accepted August 11, 2017

DOI: $10.3892 / \mathrm{ol} .2017 .7561$

\begin{abstract}
Chemotherapy is an irreplaceable treatment for prostate cancer. However, the acquisition of chemoresistance is a common and critical problem that requires urgent solutions for the effective treatment of this disease. The aim of the present study was to determine whether the combination of quercetin with doxorubicin reversed the resistance of prostate cancer cells to doxorubicin-based therapy. A prostate cancer (PC)3 cell line (PC3/R) with acquired doxorubicin-resistance was established. A significant drug-resistance to doxorubicin and high activation of the phosphoinositide 3-kinase/protein kinase-B (PI3K/AKT) pathway in PC3/R cells, compared with normal PC3 cells, was demonstrated. Notably, combination treatment of doxorubicin with quercetin significantly promoted the doxorubicin-induced apoptosis in PC3/R cells through the mitochondrial/reaction oxygen species pathway. In PC3/R cells, a significant upregulation of tyrosine-protein kinase-met (c-met) was observed compared with nromal PC3 cells. However, the response to quercetin treatment in $\mathrm{PC} 3 / \mathrm{R}$ cells inhibited c-met expression and the downstream PI3K/AKT pathway. In addition, induced expression of c-met rescued quercetin-promoted apoptosis in PC3/R cells treated with doxorubicin. The results of the present study indicated that quercetin is able to reverse prostate cancer cell doxorubicin resistance by downregulating the expression of c-met. It may represent a potential strategy for reversing the chemoresistance of prostate cancer.
\end{abstract}

\section{Introduction}

Prostate cancer is the second most common type of cancer in men, and the leading cause of cancer-associated mortality

Correspondence to: Dr Jing Chen, Department of Urology, Tongde Hospital of Zhejiang Province, 234 Gucui Road, Hangzhou, Zhejiang 310012, P.R. China

E-mail: tdjingchen@163.com

Key words: prostate cancer, doxorubicin, resistance, quercetin, tyrosine-protein kinase-Met, phosphoinositide 3-kinase/protein kinase B in men worldwide (1). Although surgery is the most effective treatment for patients with early stage prostate cancer, the tumor is unresectable for patients with advanced prostate cancer, and systemic chemotherapy is considered as the alternative option (2). However, acquired chemoresistance due to the repeated use of chemotherapeutic drugs is a common, critical problem, which limits the clinical application of chemotherapy $(3,4)$. There is an urgent requirement to develop novel strategies that may inhibit or delay the occurrence of chemoresistance.

Doxorubicin, which belongs to the family of antitumor antibiotics, is an effective type of chemotherapeutic drug. This antibiotic is able to embed into the double helical structure of cell DNA, inhibiting the synthesis of RNA and DNA, thereby inducing the apoptosis of cancer cells $(5,6)$. Although doxorubicin is widely used in the treatment of prostate cancer, there is a high rate of chemotherapeutic failure due to the acquisition of doxorubicin resistance $(7,8)$. To delay the occurrence of doxorubicin resistance, combination treatments are routinely used to offset the aforementioned drug-resistance mechanisms, and resensitize tumor cells to doxorubicin. It has been reported that curcumin improves the efficacy of doxorubicin treatment in breast cancer (9). Another study reported that the addition of microRNA-122 oligonucleotides was able to reverse doxorubicin resistance in hepatocellular carcinoma cells (10). The aforementioned studies provide evidence to demonstrate that combination treatment with other drugs are effective to inhibit or delay the occurrence of doxorubicin resistance.

Quercetin, which is widely distributed in plant-based foods, is a flavonoid. The reported pharmacological effects of quercetin include antioxidant, anti-inflammatory, and anti-proliferative activity $(11,12)$. Modern drug researchers have demonstrated that quercetin acts as a potential anti-tumor agent in multiple types of cancer by regulating the apoptosis pathway $(13,14)$. However, the anti-tumor effect of single quercetin treatment is limited. Therefore, the aim of the present study was to assess whether the combination of quercetin with doxorubicin reversed the resistance of prostate cancer cells to doxorubicin-based therapy in vitro.

\section{Materials and methods}

Cell culture. The present study was approved by the Ethics Committee of Tongde Hospital of Zhejiang (Hangzhou, China). 
The human prostate cancer cell line PC3 was obtained from the American Type Culture Collection (ATCC; Manassas, VA, USA). Doxorubicin-resistant PC3 cells (PC3/R) were established by continuous exposure of normal PC3 cells to increasing concentrations of doxorubicin (Sigma Aldrich; Merck KGaA, Darmstadt, Germany). Briefly, PC3 cells were incubated with Dulbecco's modified Eagle's medium (Gibco; Thermo Fisher Scientific, Inc., Waltham, MA, USA) supplemented with $10 \%$ fetal bovine serum (Gibco; Thermo Fisher Scientific, Inc.) and $0.05 \mu \mathrm{g} / \mathrm{ml}$ doxorubicin for 2 months. Subsequently, the dose of doxorubicin was increased every 3 weeks by $0.01 \mu \mathrm{g} / \mathrm{ml}$ up to a final concentration of $0.15 \mu \mathrm{g} / \mathrm{ml}$. Prior to the experiments, the PC3/R cells were cultured in Dulbecco's modified Eagle's medium supplemented with $10 \%$ fetal bovine serum for 2 weeks at $37^{\circ} \mathrm{C}$ in a humidified $5 \% \mathrm{CO}_{2}$ incubator.

Drug sensitivity assay. The sensitivity of PC3 and PC3/R cells to doxorubicin, and the effects of quercetin on doxorubicin-induced cell death of PC3/R cells were measured by 3-(4,5-dimethylthiazol-z-yl)-2,5-diphenyl tetrazolium bromide (MTT; Sigma Aldrich; Merck KGaA) assay. Cells were seeded into 96 -well plates at a density of $5 \times 10^{3}$ per well and cultured overnight. Cells were treated with various concentrations $(0,0.1,0.2,0.5,1.0,1.5,2,4,6,8,10$ or $15 \mathrm{~g} / \mathrm{ml})$ of doxorubicin in the presence or absence of quercetin $(10 \mu \mathrm{m}$; Sigma Aldrich; Merck KGaA) for 48 h at $37^{\circ} \mathrm{C}$. Subsequently, $20 \mu \mathrm{l}$ MTT $(5 \mathrm{mg} / \mathrm{ml})$ was added and cells were incubated at $37^{\circ} \mathrm{C}$ for an additional $4 \mathrm{~h}$. Following this, $150 \mu \mathrm{l}$ DMSO was added to dissolve the formazan crystals. The absorbance in each well was measured at $570 \mathrm{~nm}$ using a microplate reader. The half maximal inhibitory concentration $\left(\mathrm{IC}_{50}\right)$ of doxorubicin in PC3 and PC3/R cells was calculated according to the viability curves.

Western blot analysis. A total of $5 \times 10^{6}$ cells were harvested for total protein extraction and lysed with radioimmunoprecipitation assay buffer (Cell Signaling Technology, Inc., Danvers, MA, USA) for $30 \mathrm{~min}$ at $4^{\circ} \mathrm{C}$. The cell lysate $(50 \mu \mathrm{g})$ samples were separated by $10 \%$ sodium dodecyl sulfate polyacrylamide gel electrophoresis and transferred to PVDF membranes. Following transfer, membranes were incubated in $5 \%$ skimmed milk for $1 \mathrm{~h}$ at room temperature. Subsequently, the membranes were incubated with primary antibodies overnight at $4{ }^{\circ} \mathrm{C}$. Antibodys used were as follows: Anti-PI3K (cat. no. 4249; 1:1,000 dilution), anti-phosphorylated (p-) PI3K (cat. no. 13857; 1:1,000 dilution), anti-AKT (cat. no. 4685; 1:1,000 dilution), anti-p-AKT (cat. no. 4060; 1:1,000 dilution), anti-c-met (cat. no. 8198; 1:1,000 dilution), anti-caspase-9 (cat. no. 9502; 1:1,000 dilution), anti-cleaved caspase-9 (cat. no. $9505 ; 1: 1,000$ dilution), anti-caspase-3 (cat. no. 9665; 1:1,000 dilution), anti-cleaved caspase-3 (cat. no. 9664; $1: 1,000$ dilution) and anti- $\beta$-actin (cat. no. 4970; $1: 1,000$ dilution), all purchased from Cell Signaling Technology, Inc. The following day, the membranes were washed three times using Tris-buffered saline with $1 \%$ Tween-20 and incubated for $2 \mathrm{~h}$ at $37^{\circ} \mathrm{C}$ with horseradish peroxidase-conjugated goat anti-rabbit immunoglobulin G (cat no. 7074; 1:2,000 dilution; Cell Signaling Technology, Inc.). Protein bands were visualized using an enhanced chemiluminescence detection kit (Pierce; Thermo Fisher Scientific, Inc.).
Assessment of apoptosis. Cells were collected and washed with ice-cold PBS. Apoptosis was determined in PC3 and $\mathrm{PC} 3 / \mathrm{R}$ cells using a dual staining method with Annexin V-fluorescein isothiocyanate (Sigma-Aldrich; Merck KgaA) and propidium iodide for $20 \mathrm{~min}$ in the dark at room temperature. The percentage of apoptotic cells in total $10^{4}$ cells was quantified by flow cytometry (FACSCanto; BD Biosciences, Franklin Lanes, NJ, CA, USA) and analyzed by using FlowJo software (version 10; Tree Star, Inc., Ashland, OR, USA).

\section{Detection of mitochondrial membrane potential (MMP, $\Delta \Psi m$ )} and reactive oxygen species (ROS). A total of $1 \times 10^{6}$ cells were harvested and washed with ice-cold PBS. For MMP detection, cells were stained with 5,5', 6,6'-Tetrachloro-1,1',3,3'-tetraethyl imidacarbo cyanine iodide (Molecular Probes; Thermo Fisher Scientific, Inc.) for $15 \mathrm{~min}$ in the dark at room temperature. For measurement of ROS, cells were stained with dihydroethidium (Molecular Probes; Thermo Fisher Scientific, Inc.) for $15 \mathrm{~min}$ in the dark at room temperature. Detection of MMP and ROS were analyzed by flow cytometry (FACSCanto; BD Biosciences) and FlowJo 10 software.

Recombinant plasmid construction and transfection. Total RNAs from PC3 cells were extracted using TRIzol reagent (Invitrogen; Thermo Fisher Scientific, Inc.) according to the manufacturer's protocol. Total RNA was reverse transcribed into cDNA using the Prime Script RT reagent kit (Takara Biotechnology Co., Ltd. Dailan, China) following the manufacturer's protocol. Following synthesis of PC3 cDNA, the open reading frame of the c-met gene was amplified by polymerase chain reaction (PCR) using Takara LA Taq ${ }^{\circledR}$ (Takara Biotechnology Co., Ltd.) using the following primers: Forward, c-met HindIII, 5'-ACGAAGCTTATGAAGGCC CCCGCTGTGCTT-3', and reverse, c-met XhoI, ACGCTC GAGTGATGTCTCCCAGAAGGAGGCTGGT. The reaction conditions were as follows: $94^{\circ} \mathrm{C}$ for $1 \mathrm{~min}, 30$ cycles of $98^{\circ} \mathrm{C}$ for $10 \mathrm{sec}, 68^{\circ} \mathrm{C}$ for $5 \mathrm{~min}$ and $72^{\circ} \mathrm{C}$ for $10 \mathrm{~min}$. PCR products and the pcDNA3.1 plasmid (Invitrogen; Thermo Fisher Scientific, Inc.) were digested with HindIII/XhoI (Takara Biotechnology Co., Ltd.) for $2 \mathrm{~h}$ at $37^{\circ} \mathrm{C}$. To ligate the c-met fragment into the pcDNA3.1 plasmid, the digestion products were incubated with T4 DNA ligase (Takara Biotechnology Co., Ltd.) overnight at $16^{\circ} \mathrm{C}$ and the recombinant plasmid was named the c-met vector. For transfection, the $5 \times 10^{5} \mathrm{PC} 3 / \mathrm{R}$ cells were plated and cultured to reach $80 \%$ confluency. C-met vector $(2 \mu \mathrm{g} / \mathrm{ml})$ or empty vector (used for control) was transiently transfected into the cells with Lipofectamine 2000 reagent (Invitrogen; Thermo Fisher Scientific, Inc.) according to the the manufacturer's protocol.

Statistical analysis. Statistical analyses were conducted on all the experiments, which were repeated in triplicate and data were expressed as the mean \pm standard deviation. For comparison analysis, two-tailed unpaired Student's t-test was used to evaluate the statistical differences between two groups. One-way analysis of variance and Bonferroni's post-hoc test were used to determine the differences between three or more groups. Statistical analysis was performed using SPSS software (version 15.0; SPSS, Inc., Chicago, IL, USA). P<0.05 was considered to indicate a statistically significant difference. 

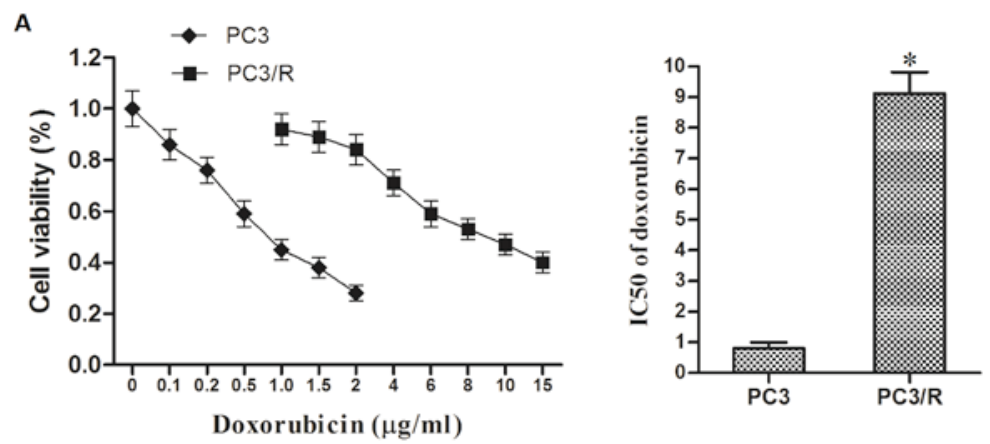

B
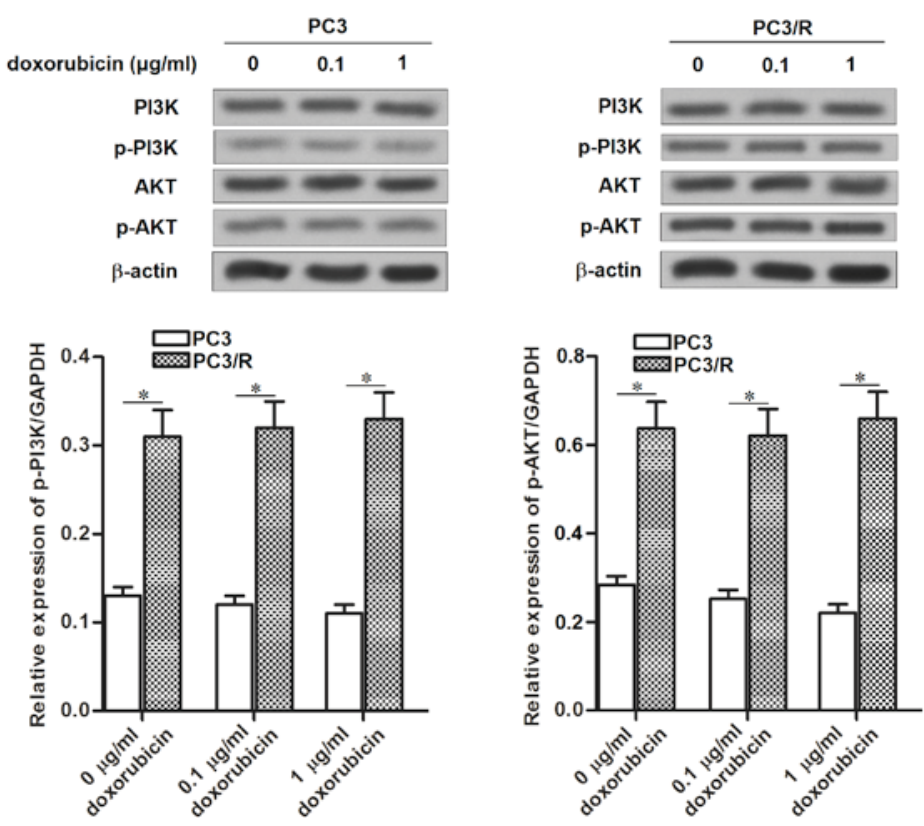

Figure 1. Comparison of doxorubicin sensitivity between PC3/R and PC3 cell lines. (A) Following treatment with doxorubicin at the indicated concentrations for $48 \mathrm{~h}$, the cell viability of PC $3 / \mathrm{R}$ and PC3 were detected by MTT assay. The $\mathrm{IC}_{50}$ of PC3/R and PC3 to doxorubicin was calculated according to the viability curves. (B) Following $48 \mathrm{~h}$ of doxorubicin treatment, the activation of PI3K/AKT pathway was evaluated by western blot analysis in PC3/R and PC3 cells ${ }^{*} \mathrm{P}<0.05$ vs. PC3 cell line. PC3/R, prostate cancer 3/resistant; PC3, prostate cancer 3; MTT, 3-(4,5-dimethylthiazol-2-yl)-2,5-diphenyltetrazolium bromide; $\mathrm{IC}_{50}$, half maximal inhibitory concentration. P13K, phosphoinositide 3-kinase; AKT, protein kinase B; P-, phosphorylated.

\section{Results}

Resistance of PC3/R cells to doxorubicin. To investigate the resistance of $\mathrm{PC} 3$ prostate cancer cells to doxorubicin, a PC3/R cell line was established by continuous exposure of routine PC3 cells to doxorubicin. The results presented in Fig. 1A demonstrated that $\mathrm{IC}_{50}$ of doxorubicin to $\mathrm{PC} 3 / \mathrm{R}$ was 11.25 -fold higher than the parental PC 3 cells. This indicated that the established $\mathrm{PC} 3 / \mathrm{R}$ cell line demonstrated significant drug resistance to doxorubicin. Due to data published from previous studies, which provided evidence that the PI3K/AKT pathway regulates the chemotherapeutic resistance of cells $(15,16)$, the activation of PI3K and AKT in PC3/R cells compared with parental PC3 cells, in response to doxorubicin, was investigated. Notably, activation of the PI3K/AKT pathway was significantly increased in PC3/R cells compared with parental PC3 cells, in the presence or absence of equal doses of doxorubicin (Fig. 1B). The results from the present study suggested that the hyper-activation of the PI3K/AKT pathway may be responsible for the drug resistance to doxorubicin in PC $3 / \mathrm{R}$ cells.

Quercetin increased the sensitivity of PC3/R cells to doxorubicin. To investigate the effect of quercetin on doxorubicin resistance, PC3/R cells were co-treated with doxorubicin and quercetin. Analysis of cell viability revealed that although single quercetin treatment alone had no effect on the viability of PC3/R cells, in combination with doxorubicin treatment, quercetin significantly enhanced the effects of doxorubicin and reduced $\mathrm{PC} 3 / \mathrm{R}$ cell viability compared with cells treated with doxorubicin alone (Fig. 2A). In addition, the results of flow cytometry indicated that the combination of quercetin and doxorubicin induced significantly increased apoptosis in PC3/R cells compared with cells treated with doxorubicin alone (Fig. 2B). Taken together, these results demonstrated that quercetin in combination with doxorubicin was able to reverse drug resistance in doxorubicin resistant prostate cancer cells.

Combination treatment with quercetin and doxorubicin induced apoptosis via the mitochondrial pathway. To investigate the mechanism by which quercetin promoted doxorubicin-induced apoptosis, MMP was measured in $\mathrm{PC} 3 / \mathrm{R}$ cells, co-treated with quercetin and doxorubicin. The results presented in Fig. 3A demonstrated that combination treatment with quercetin and doxorubicin induced a significant decrease of MMP in PC3/R cells compared with cells treated with doxorubicin alone. Furthermore, ROS, 


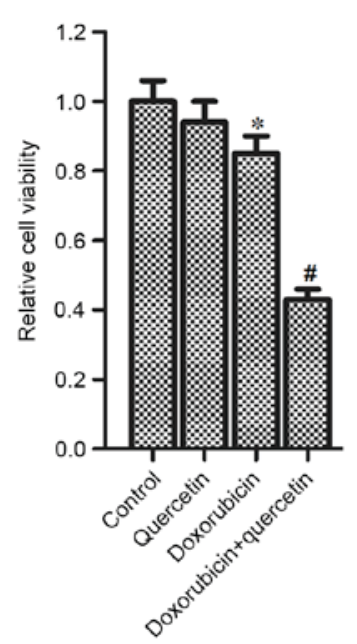

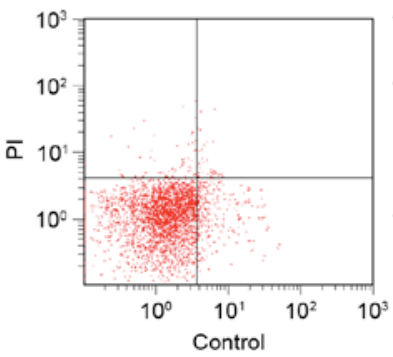

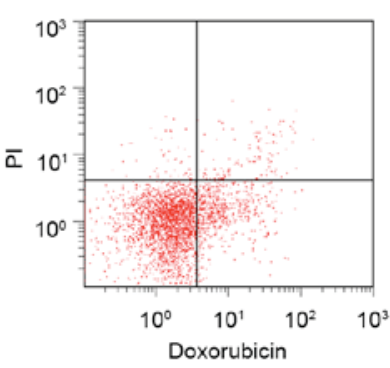

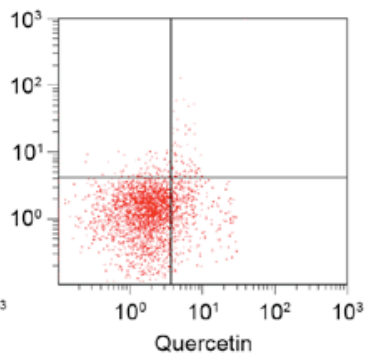

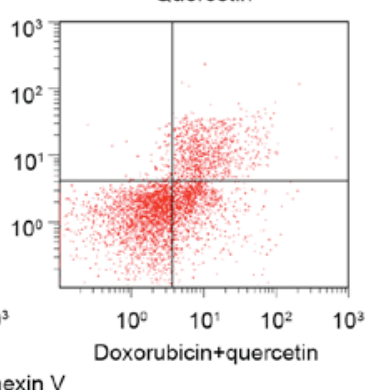

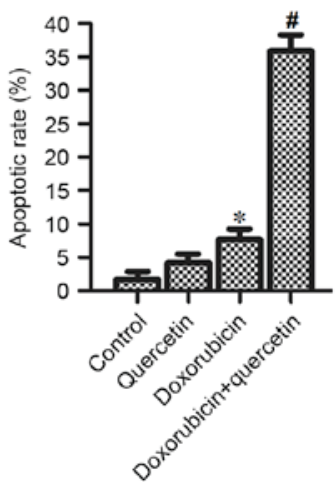

Figure 2. Quercetin enhanced the cytotoxicity of doxorubicin to PC3/R. (A) Following treatment with quercetin $(10 \mu \mathrm{M})$ and doxorubicin $(2 \mu \mathrm{g} / \mathrm{ml})$ for $48 \mathrm{~h}$ the relative cell viability of PC3/R and PC 3 cells was determined by MTT assay. (B) Following treatment with quercetin (10 $\mu \mathrm{M})$ and doxorubicin (2 $\mu \mathrm{g} / \mathrm{ml})$ in $\mathrm{PC} 3 / \mathrm{R}$ and PC3 cells for $48 \mathrm{~h}$, cell apoptosis was detected by flow cytometry. ${ }^{~} \mathrm{P}<0.05$ vs. control group, ${ }^{*} \mathrm{P}<0.05$ vs. doxorubicin group. PC3/R, prostate cancer 3/resistant; PC3, prostate cancer 3; PI, propidium iodide.

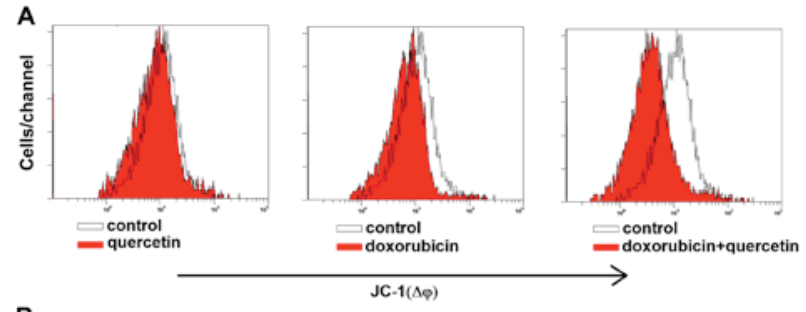

B

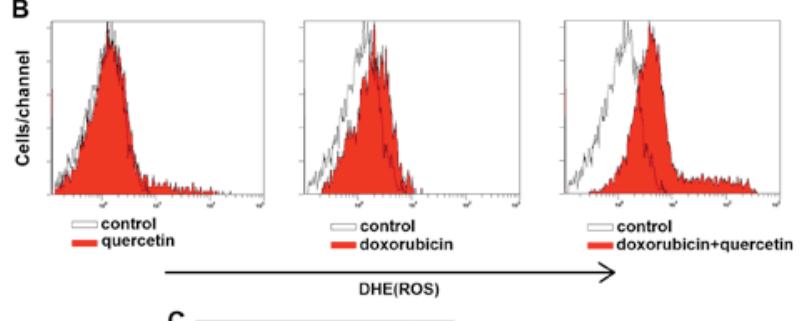

C

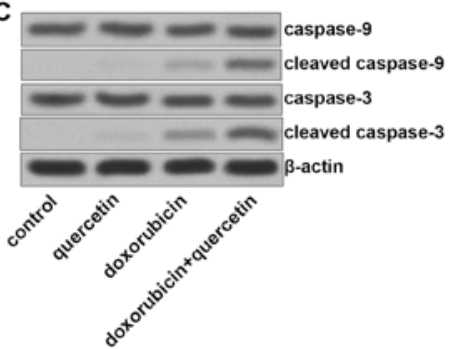

Figure 3. Quercetin promoted doxorubicin-induced apoptosis via the mitochondrial pathway. (A) The mitochondrial membrane potential of PC3/R cells treated with quercetin $(10 \mu \mathrm{M})$ and doxorubicin $(2 \mu \mathrm{g} / \mathrm{ml})$ was measured by JC-1 staining and flow cytometry. (B) The generation of ROS in PC3/R cells treated with quercetin $(10 \mu \mathrm{M})$ and doxorubicin $(2 \mu \mathrm{g} / \mathrm{ml})$ was measured by DHE staining and flow cytometry. (C) Western blot analysis was performed to evaluate the cleavage of caspase- 9 and caspase- 3 in the PC $3 / \mathrm{R}$ cells treated with quercetin $(10 \mu \mathrm{M})$ and doxorubicin $(2 \mu \mathrm{g} / \mathrm{ml})$. PC3/R, prostate cancer 3/resistant; ROS, reactive oxygen species; DHE, dihydroethidium.

which are considered to be key apoptotic inducers (17) were released from the mitochondria into the cytoplasm, due to
MMP collapse induced by co-treatment with quercetin and doxorubicin (Fig. 3B). The expression of caspase-3 and -9 in response to quercetin and doxorubicin treatment were further investigated, as these are known to be activated downstream of the mitochondrial pathway (Fig. 3C). The effects of doxorubicin treatment were enhanced by combination treatment with quercetin to induce expression of cleaved caspase- 3 and -9. The results from the present study therefore demonstrated that quercetin promoted doxorubicin-induced apoptosis via the mitochondrial pathway.

$C$-met is the target of quercetin in PC3/R cells. The results from the present study have indicated thus far that PC3/R cells demonstrate hyper-activation of PI3K/AKT compared with normal PC3 cells. To elucidate how quercetin facilitates doxorubicin-induced cell death in PC3/R cells, the target of quercetin in the PI3K/AKT pathway in PC3/R cells was explored. The expression level of c-met, which is upstream of PI3K signaling (18), was significantly increased in PC3/R cells compared with in PC3 cells (Fig. 4A). Thus, hyper-activation of the c-met/PI3K/AKT pathway may be responsible for doxorubicin resistance in PC $3 / \mathrm{R}$ cells. However, treatment with quercetin without doxorubicin significantly downregulated c-met expression compared to the control group (Fig. 4B). In addition, the activation of the PI3K/AKT pathway was also inhibited by quercetin treatment. Furthermore, the induced expression of c-met abolished the inhibition of the PI3K/AKT pathway induced by quercetin (Fig. 4C). Taken together, the results revealed that quercetin targeted c-met to inhibit the $\mathrm{PI} 3 \mathrm{~K} / \mathrm{AKT}$ pathway in doxorubicin-resistant prostate cancer cells.

Quercetin increased the sensitivity of PC3/R to doxorubicin via downregulating $c$-met expression. To study the function of c-met and the synergistic effects of quercetin and 


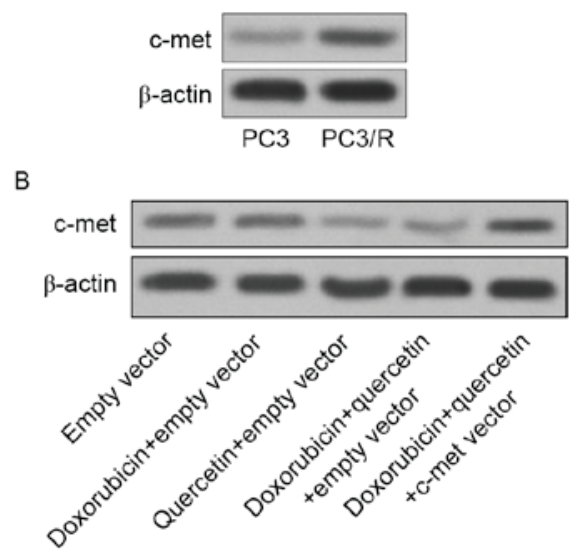

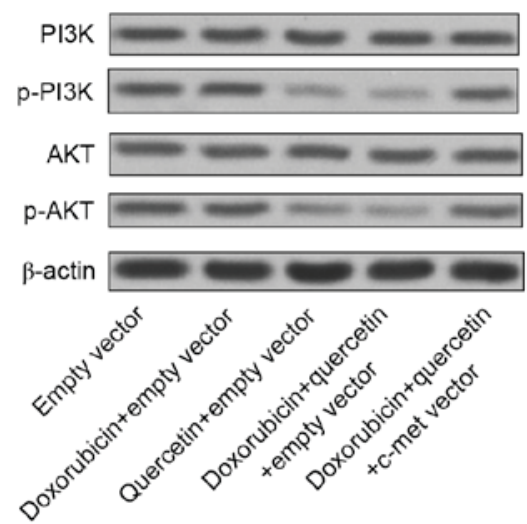

Figure 4. C-met is the target of quercetin in PC3/R. (A) Expression of c-met in PC3/R and PC3 cells was evaluated by western blotting. (B) Expression of c-met was analyzed by western blotting in PC3/R cells transfected with an empty vector or c-met vector, and treated with quercetin $(10 \mu \mathrm{M})$ and doxorubicin $(2 \mu \mathrm{g} / \mathrm{ml})$. (C) The activation of P13K and AKT was evaluated by western blotting in PC3/R cells transfected with empty vector or c-met vector, and treated with quercetin $(10 \mu \mathrm{M})$ and doxorubicin $(2 \mu \mathrm{g} / \mathrm{ml})$. C-met, tyrosine-protein kinase-Met; PC3/R, prostate cancer 3/resistant; P13K, phosphoinositide 3-kinase; AKT, protein kinase-B; P-, phosphorylated.

A

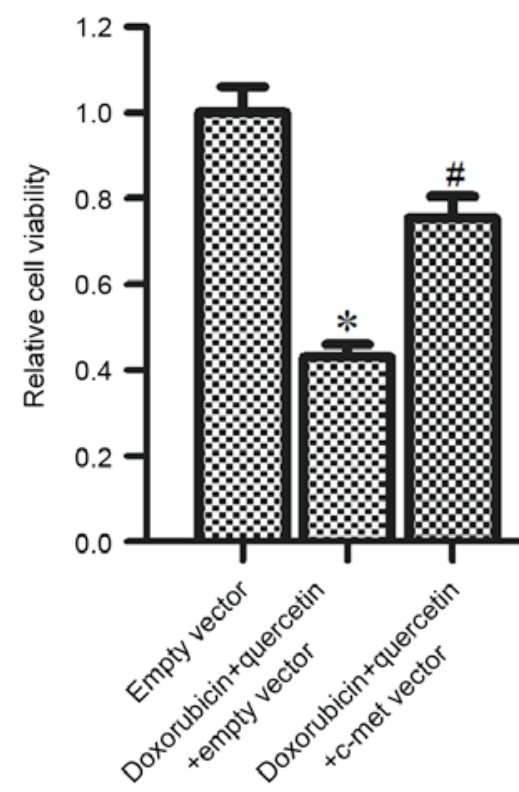

B
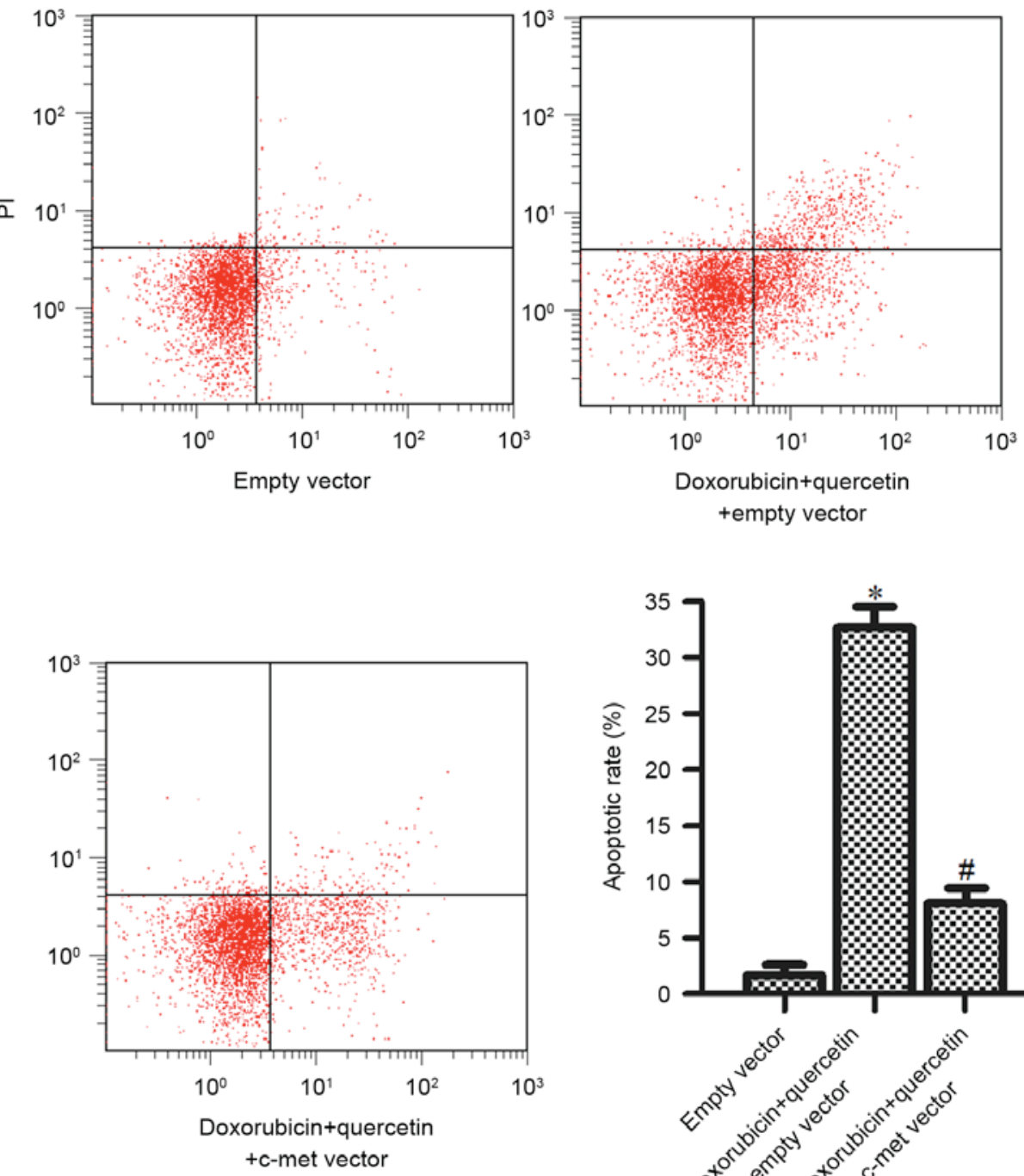

Annexin V

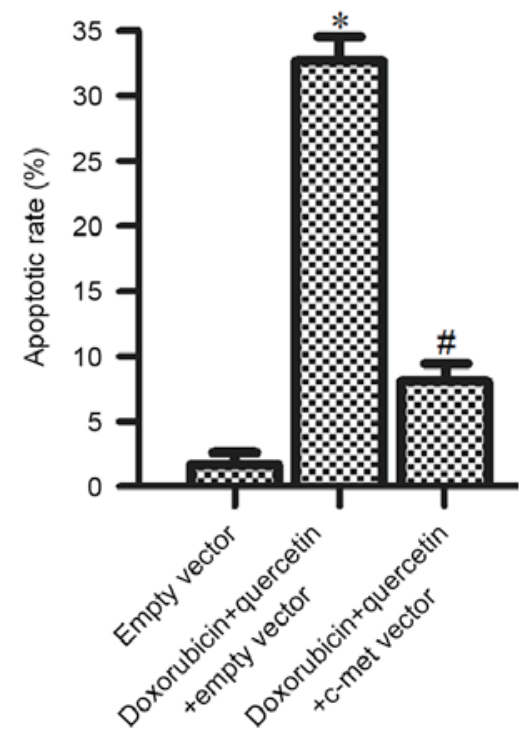

Figure 5. Quercetin increases the sensitivity of PC3/R to doxorubicin via downregulating c-met expression. (A) Cell viability was measured by MTT assay in PC3/R cells transfected with empty vector or c-met vector, and treated with quercetin $(10 \mu \mathrm{M})$ and doxorubicin $(2 \mu \mathrm{g} / \mathrm{ml})$. (B) Cell apoptosis was determined by flow cytometry. ${ }^{~} \mathrm{P}<0.05$ vs. control group, ${ }^{,} \mathrm{P}<0.05$ vs. doxorubicin + quercetin group. $\mathrm{PC} 3 / \mathrm{R}$, prostate cancer $3 /$ resistant; c-met, tyrosine-protein kinase-Met. 
doxorubicin, c-met expression was induced in PC3/R cells to rescue cell death induced by this combined treatment. It was observed that introduction of the c-met vector significantly inhibited the cytotoxicity, induced in cells by the combination treatment with quercetin and doxorubicin (Fig. 5A). Furthermore, quercetin and doxorubicin induced-apoptosis was also inhibited by the c-met vector (Fig. 5B). The results from the present study suggested that the synergistic effects of quercetin and doxorubicin were dependent on the downregulation of c-met.

\section{Discussion}

C-met is the receptor for hepatocyte growth factor (HGF). The extracellular sema domain of c-met mediates binding to HGF, which activates receptor auto-phosphorylation (19). The activation of c-met has been reported to stimulate multiple downstream genes to promote tumorigenesis in various types of cancer (20). Overexpression of c-met stimulates proliferation, migration and invasion in various types of cancer including prostate cancer $(21,22)$. Furthermore, activation of the c-met pathway was revealed to be an important mechanism for acquired resistance to chemotherapy. Previous studies have reported that $\mathrm{c}$-met receptor activation protects cancer cells against DNA-damaging agents. For example, activation of the c-met pathway triggered the expression of focal adhesion kinase and downregulated apoptosis-inducing factor expression to develop cisplatin resistance in lung cancer (23). In human multiple myeloma, c-met knockdown resulted in decreased drug-resistance and increased chemosensitivity to doxorubicin (24). Therefore, the inhibition of c-met and its downstream signaling targets has been considered as a potential strategy to enhance the therapeutic efficacy for the treatment of cancer $(25,26)$.

Within the c-met pathway, the auto-phosphorylation of c-met leads to phosphorylation of PI3K and the subsequent generation of phosphatidylinositol-3,4,5-trisphosphate (PIP3). Then, cellular PIP3 triggers AKT activation $(27,28)$. It has been reported that PI3K/AKT signaling stimulates various biological processes in cancer, and functions as a key regulator in cell proliferation, survival, migration and apoptosis $(29,30)$. Hyper-activation of the PI3K/AKT pathway is observed in several types of cancer, such as colorectal (31), ovarian (32) and prostate cancer (33). Previous studies have revealed a direct link between highly activated PI3K/AKT and poor prognosis and tumor recurrence in prostate cancer (33). In addition, as the PI3K/AKT pathway regulates the apoptosis of cancer cells, it accelerates chemoresistance in cancer cells when under the influence of chemotherapeutic drugs (34). Therefore, intervention of this pathway is considered to be a potential strategy to delay or reverse the occurrence of drug resistance in cancer.

Quercetin is a natural flavonoid compound. Previous studies have demonstrated that quercetin is involved in inducing the apoptosis pathway in cancers (35). Therefore, combination treatment with quercetin and several anti-tumor agents, including docetaxel, tumor necrosis factor-related apoptosis-inducing ligand, and 2-methoxyestradiol have proved to be effective synergistic treatments for prostate cancer (36-38). The fact that doxorubicin treatment in prostate cancer frequently leads to the acquirement of doxorubicin resistance is a major problem. To study the potential function of quercetin in doxorubicin resistance in prostate cancer, a doxorubicin-resistant PC3 cell line PC3/R was established. Notably, it was observed that the addition of quercetin was able to reverse doxorubicin resistance, by increasing the sensitivity of PC3/R cells to doxorubicin-induced apoptosis in vitro.

The activated c-met/PI3K/AKT pathway protects cancer cells from apoptotic signals and promotes cancer survival (39). In the present study, it was revealed that c-met was further upregulated in response to the acquisition of doxorubicin-resistance in prostate cancer cells. Therefore, it is suggested that c-met is associated with drug-resistance in prostate cancer. Furthermore, it was demonstrated that quercetin treatment significantly inhibited c-met expression in PC3/R cells. Subsequently, the inhibition of the PI3K/AKT pathway, which is downstream of c-met, was also observed. Furthermore, as the combination treatment with quercetin inhibited the PI3K/AKT pathway, doxorubicin-induced dysfunction of mitochondria, release of ROS, cleavage of caspase-3/9 and apoptosis in PC3/R cells occurred as a consequence. Additionally, quercetin-induced doxorubicin cytotoxicity in PC3/R cells was impaired in response to the overexpression of $\mathrm{c}$-met, induced by its expression plasmid. Taken together, these results provide evidence that combination treatment with quercetin is able to reverse doxorubicin-resistance in prostate cancer cells by targeting the c-met/PI3K/AKT pathway. In conclusion, the present study may provide a novel treatment protocol for inhibiting or delaying drug resistance to doxorubicin-based therapy.

\section{References}

1. Siegel R, Naishadham D and Jemal A: Cancer statistics, 2013. CA Cancer J Clin 63: 11-30, 2013.

2. Kopczyńska E: Role of microRNAs in the resistance of prostate cancer to docetaxel and paclitaxel. Contemp Oncol (Pozn) 19: 423-427, 2015.

3. Zhang W, Meng Y, Liu N, Wen XF and Yang T: Insights into chemoresistance of prostate cancer. Int J Biol Sci 11: 1160-1170, 2015.

4. Karnak D and $\mathrm{Xu} \mathrm{L}$ : Chemosensitization of prostate cancer by modulating Bcl-2 family proteins. Curr Drug Targets 11: 699-707, 2010.

5. He H, Tian W, Chen H and Deng Y: MicroRNA-101 sensitizes hepatocellular carcinoma cells to doxorubicin-induced apoptosis via targeting Mcl-1. Mol Med Rep 13: 1923-1929, 2016.

6. Rivankar S: An overview of doxorubicin formulations in cancer therapy. J Cancer Res Ther 10: 853-858, 2014.

7. De Luca P, Vazquez ES, Moiola CP, Zalazar F, Cotignola J, Gueron G, Gardner K and De Siervi A: BRCA1 loss induces GADD153-mediated doxorubicin resistance in prostate cancer. Mol Cancer Res 9: 1078-1090, 2011.

8. Carey JP, Knowell AE, Chinaranagari S and Chaudhary J: Id4 promotes senescence and sensitivity to doxorubicin-induced apoptosis in DU145 prostate cancer cells. Anticancer Res 33: 4271-7278, 2013.

9. Lv L, Qiu K, Yu X, Chen C, Qin F, Shi Y, Ou J, Zhang T, Zhu H, Wu J, et al: Amphiphilic Copolymeric micelles for doxorubicin and curcumin co-delivery to reverse multidrug resistance in breast cancer. J Biomed Nanotechnol 12: 973-985, 2016.

10. Pan C, Wang X, Shi K, Zheng Y, Li J, Chen Y, Jin L and Pan Z: MiR-122 reverses the doxorubicin-resistance in hepatocellular carcinoma cells through regulating the tumor metabolism. PLoS One 11: e0152090, 2016.

11. Bischoff SC: Quercetin: Potentials in the prevention and therapy of disease. Curr Opin Clin Nutr Metab Care 11: 733-740, 2008.

12. Erlund I: Review of the flavonoids quercetin, hespertin and naringenin. dietary sources, bioactivities, bioavailability and epidemiology. Nutr Res 24: 851-874, 2004. 
13. Ramos S: Effects of dietary flavonoids on apoptotic pathways related to cancer chemoprevention. J Nutr Biochem 18: 427-442, 2007.

14. Li J, Tang C, Li L, Li R and Fan Y: Quercetin sensitizes glioblastoma to $\mathrm{t}$-AUCB by dual inhibition of Hsp27 and COX-2 in vitro and in vivo. J Exp Clin Cancer Res 35: 61, 2016.

15. West KA, Castillo SS and Dennis PA: Activation of the PI3K/Akt pathway and chemotherapeutic resistance. Drug Resist Updat 5: 234-248, 2002.

16. Kang Q and Yan S: Piperlongumine reverses doxorubicin resistance through the PI3K/Akt signaling pathway in K562/A02 human leukemia cells. Exp Ther Med 9: 1345-1350, 2015.

17. Zorov DB, Juhaszova M and Sollott SJ: Mitochondrial reactive oxygen species (ROS) and ROS-induced ROS release. Physiol Rev 94: 909-950, 2014.

18. Jung KA, Choi BH and Kwak MK: The c-MET/PI3K signaling is associated with cancer resistance to doxorubicin and photodynamic therapy by elevating BCRP/ABCG2 expression. Mol Pharmacol 87: 465-476, 2015.

19. Ferracini R, Longati P, Naldini L, Vigna E and Comoglio PM: Identification of the major autophosphorylation site of the Met/hepatocyte growth factor receptor tyrosine kinase. J Biol Chem 266: 19558-19564, 1991.

20. Knudsen BS and Vande Woude G: Showering c-Met dependent cancers with drugs. Curr Opin Genet Dev 18: 87-96, 2008.

21. Han Y, Luo Y, Zhao J, Li M and Jiang Y: Overexpression of c-Met increases the tumor invasion of human prostate LNCaP cancer cells in vitro and in vivo. Oncol Lett 8: 1618-1624, 2014.

22. Yasuda K, Nagakawa O, Akashi T, Fujiuchi Y, Koizumi K, Komiya A, Saiki I and Fuse H: Serum active hepatocyte growth factor (AHGF) in benign prostatic disease and prostate cancer. Prostate 69: 346-351, 2009.

23. Chen JT, Huang CY, Chiang YY, Chen WH, Chiou SH, Chen CY and Chow KC: HGF increases cisplatin resistance via down-regulation of AIF in lung cancer cells. Am J Respir Cell Mol Biol 38: 559-565, 2008.

24. Que W and Chen J: Knockdown of c-Met inhibits cell proliferation and invasion and increases chemosensitivity to doxorubicin in human multiple myeloma U266 cells in vitro. Mol Med Rep 4: 343-349, 2011.

25. Toschi L and Jänne PA: Single-agent and combination therapeutic strategies to inhibit hepatocyte growth factor/MET signaling in cancer. Clin Cancer Res 14: 5941-5946, 2008.

26. Hung TH, Li YH, Tseng CP, Lan YW, Hsu SC, Chen YH, Huang TT, Lai HC, Chen CM, Choo KB and Chong KY: Knockdown of c-MET induced apoptosis in ABCB1-overexpressed multidrug-resistance cancer cell lines. Cancer Gene Ther 22: 262-270, 2015.

27. Yao Y, Dou C, Lu Z, Zheng X and Liu Q: MACC1 suppresses cell apoptosis in hepatocellular carcinoma by targeting the HGF/c-MET/AKT pathway. Cell Physiol Biochem 35: 983-996, 2015.
28. Trovato M, Torre ML, Ragonese M, Simone A, Scarfì R, Barresi V, Giuffrè G, Benvenga S, Angileri FF, Tuccari G, et al: HGF/c-met system targeting PI3K/AKT and STAT3/phosphorylated-STAT3 pathways in pituitary adenomas: an immunohistochemical characterization in view of targeted therapies. Endocrine 44: 735-743, 2013.

29. Engelman JA: Targeting PI3K signalling in cancer: Opportunities, challenges and limitations. Nat Rev Cancer 9: 550-562, 2009.

30. Jiang BH and Liu LZ: PI3K/PTEN signaling in angiogenesis and tumorigenesis. Adv Cancer Res 102: 19-65, 2009.

31. Danielsen SA, Eide PW, Nesbakken A, Guren T, Leithe E and Lothe RA: Portrait of the PI3K/AKT pathway in colorectal cancer. Biochim Biophys Acta 1855: 104-121, 2015.

32. Li H,Zeng J and Shen K: PI3K/AKT/mTOR signaling pathway as a therapeutic target for ovarian cancer. Arch Gynecol Obstet 290: 1067-1078, 2014

33. Toren $\mathrm{P}$ and Zoubeidi A: Targeting the PI3K/Akt pathway in prostate cancer: Challenges and opportunities (review). Int $\mathbf{J}$ Oncol 45: 1793-1801, 2014.

34. Tang KD and Ling MT: Targeting drug-resistant prostate cancer with dual PI3K/mTOR inhibition. Curr Med Chem 21: 3048-3056, 2014

35. Yang F, Song L, Wang H, Wang J, Xu Z and Xing N: Quercetin in prostate cancer: Chemotherapeutic and chemopreventive effects, mechanisms and clinical application potential (Review). Oncol Rep 33: 2659-2668, 2015.

36. Wang P, Henning SM, Magyar CE, Elshimali Y, Heber D and Vadgama JV: Green tea and quercetin sensitize PC-3 xenograft prostate tumors to docetaxel chemotherapy. J Exp Clin Cancer Res 35: 73, 2016

37. Jung YH, Heo J, Lee YJ, Kwon TK and Kim YH: Quercetin enhances TRAIL-induced apoptosis in prostate cancer cells via increased protein stability of death receptor 5. Life Sci 86: 351-357, 2010.

38. Yang F, Song L, Wang H, Wang J, Xu Z and Xing N: Combination of quercetin and 2-Methoxyestradiol enhances inhibition of human prostate cancer LNCaP and PC-3 cells xenograft tumor growth. PLoS One 10: e0128277, 2015.

39. Jiao D, Wang J, Lu W, Tang X, Chen J, Mou H and Chen QY: Curcumin inhibited HGF-induced EMT and angiogenesis through regulating c-Met dependent PI3K/Akt/mTOR signaling pathways in lung cancer. Mol Ther Oncolytics 3: 16018, 2016.

This work is licensed under a Creative Commons Attribution-NonCommercial-NoDerivatives 4.0 International (CC BY-NC-ND 4.0) License. 